\title{
Miser sur l'engagement mutuel pour contribuer au développement professionnel continu : le modèle d'une communauté de pratique au préscolaire (COPP)
}

\author{
Hélène Larouche, Diane Biron, Julie Vaillancourt
}

\begin{abstract}
Hélène Larouche $(\mathbf{P h}$. D.) est professeure au Département de l'enseignement au préscolaire et au primaire de la Faculté d’éducation l'Université de Sherbrooke. Ses domaines de spécialisation sont, principalement, l'éducation préscolaire, le développement professionnel des enseignants et l'intervention pédagogique. Ses thèmes de recherche collaborative portent, entre autres, sur les récits de pratique des enseignants, les pratiques favorisant l'apprentissage actif chez les enfants de maternelle et sur la reconnaissance du savoir d'expérience des praticiens.
\end{abstract}

Diane Biron (Ph. D.) est professeure au Département de l'enseignement au préscolaire et au primaire de la Faculté d’éducation de l'Université de Sherbrooke. Son domaine de spécialisation est la didactique des mathématiques et ses principaux thèmes de recherche portent sur le développement de la pensée mathématique chez l'enfant de 4 à 8 ans ainsi que sur le rôle du jeu, du questionnement et de la manipulation dans l'apprentissage des mathématiques. De plus, elle s'intéresse à l'apport de la littérature jeunesse dans une perspective interdisciplinaire, au processus de résolution de problèmes et aux pratiques qui favorisent l'apprentissage actif de l'enfant.

Julie Vaillancourt (B. Éd.) est étudiante à la maîtrise en enseignement au préscolaire et au primaire à l'Université de Sherbrooke. Après l'obtention de son baccalauréat en enseignement au préscolaire et au primaire, elle a choisi de poursuivre ses études au deuxième cycle afin de mieux comprendre comment une enseignante de maternelle peut soutenir et enrichir le jeu des enfants. Présentement, ses intérêts de recherche portent principalement sur les interventions de l'adulte à l'éducation préscolaire.

Cet article traite d'une démarche de développement professionnel continu fondée sur la théorie des communautés de pratique de Wenger (2005). Elle s'est déroulée sur trois ans auprès de douze enseignantes du préscolaire de la région de Sherbrooke au Québec. Le concept d'engagement est approfondi en tenant compte d'un point de vue individuel et collectif afin de documenter une culture de collaboration. Les dispositifs mis en place sont précisés ainsi que les forces et les limites observées. Les données collectées, par observations, par comptes rendus, par questionnaires et par entretiens individuels permettent de dégager a posteriori certains constats surlesmoments porteursetrassembleurs de la démarche ainsi que sur les moments critiques qui peuvent influencer l'engagement des enseignantes et leur participation au sein d'une communauté de pratique.

Key words: développement professionnel continu, communauté de pratique, préscolaire, apprentissage actif
This article discusses an ongoing professional development approach based on Wenger's Community of Practice Theory (2005). It took place over three years with twelve preschool teachers from the Sherbrooke region of Quebec. The concept of commitment is deepened by taking into account an individual and collective point of view in order to document a culture of collaboration. The devices set up are specified as well as the forces and limits observed. The data collected, by observations, by reports, by questionnaires and by individual interviews, provide a posteriori with some observations on the carrying and gathering moments of the approach as well as on the critical moments can influence the commitment of teachers and their participation in a community of practice.

Key words: Continuing professional development, community of practice, preschool, active learning 


\section{Miser sur l'engagement mutuel pour contribuer au développement professionnel continu : le modèle d'une communauté de pratique au préscolaire (CoPP)}

Dans cet article, une démarche de recherche-formation menée en collaboration entre les milieux scolaire et universitaire est décrite. D’entrée de jeu, la problématique expose les principales embûches qui entravent la formation continue offerte aux enseignants et enseignantes. Elle s'attarde de manière plus spécifique sur ce qui est souvent perçu comme un manque de pertinence des pratiques de développement professionnel continu. Celui-ci est examiné sous l'angle du "Long Life Learning ", soit l'apprentissage tout au long de la vie (UNESCO, 2015). Une fois le problème posé, l'étude propose une perspective prometteuse : miser sur l'engagement afin de favoriser une culture de collaboration. Le cadre conceptuel, pour sa part, puise ses fondements dans les travaux de Wenger (2005) sur les communautés de pratique (CoP). Vient à sa suite la méthodologie de la recherche qui poursuit l'objectif suivant : identifier les dispositifs favorisant l'engagement des enseignantes participantes à la démarche de développement professionnel continu. Enfin, les résultats apportent un éclairage sur les moments porteurs et rassembleurs ainsi que les moments critiques liés à l'engagement afin de documenter les conditions favorisant une culture de collaboration au sein d'une CoP.

\section{La problématique : explorer de nouvelles avenues en formation continue}

Lévolution de la société québécoise a conduit le ministère de l'Éducation du Loisir et du Sport du Québec (Gouvernement du Québec, 2002) à élaborer, au début des années 2000, la Politique gouvernementale de léducation des adultes et de formation continue qui rejoint le paradigme du «Long, Life, Learning », soit l'apprentissage tout au long de la vie dont fait mention l'UNESCO (2015). Dans une approche résolument centrée sur la personne, il est recommandé de miser sur des modèles partenariaux pour accroître et valoriser les compétences professionnelles (Gouvernement du Québec, 2002). Parmi tous les acteurs visés, un accent est mis sur les enseignantes et enseignants afin de faciliter et de rendre accessible la formation continue. Cette politique a invité explicitement les établissements scolaires à assurer ce type de service. Par ailleurs, une récente étude canadienne montre que $90 \%$ des enseignantes et enseignants sont engagés dans différentes formes de formation professionnelle continue (Campbell, OsmondJohnson et Faubert, 2016). Lobjectif étant ici non pas d'uniformiser les approches ni de les comparer entre elles, mais bien de comprendre et de valoriser les différentes expériences d'apprentissage professionnel dans les provinces et territoires canadiens. Parmi les résultats, certains éléments communs sont associés à l’apprentissage professionnel efficace, dont le besoin d'expériences d’apprentissage pertinentes, pratiques et collaboratives à l'école et à l'extérieur de l'école.

Plusieurs écrits soulignent que la formation professionnelle continue offerte au personnel enseignant ne correspond pas toujours à leurs besoins (Beaumont, Lavoie et Couture, 2001; CSÉ, 2014; Hargreaves et Fullan, 2013 ; Helterbran et Fennimore, 2004 ; Li, 2008). Les enseignants et enseignantes repartent des journées de perfectionnement remplis de bonnes intentions par rapport à des contenus diversifiés mais, de retour en classe, la mise en œuvre de nouvelles 
pratiques est vite dépassée par la réalité et les défis quotidiens (Beaumont, Lavoie et Couture, 2001). Il y a donc lieu de se questionner sur les approches favorables au développement professionnel des enseignantes et enseignants. Selon la définition adoptée par le Conseil supérieur de léducation (CSÉ, 2014), le développement professionnel englobe la formation initiale, l'insertion professionnelle et la formation continue. C'est principalement sur cette dernière étape du continuum que porte le projet du présent article qui concerne essentiellement des enseignantes de maternelle. Ce niveau correspond à des besoins exprimés dans les milieux scolaires et à nos champs d’expertise comme chercheuses.

Une des priorités identifiées dans l'étude de Campbell et ses collègues (2016) indique que les enseignantes et enseignants souhaitent mieux comprendre comment soutenir les divers besoins de leurs élèves. Dans les composantes clés du développement professionnel, ces auteurs soulignent certains aspects structurels, dont une approche personnalisée où la collaboration et la mise à profit de l'expérience sont privilégiées. Il apparaît aussi qu'une des conditions essentielles d'un changement effectif de pratique réside dans les interactions entre pairs : «la valeur de l'expérience partagée entre enseignants constitue un des outils les plus puissants en terme d'apprentissage entre pairs et donc de développement des capacités professionnelles » (Rey, 2016, p. 10).

De notre point de vue, la formation continue se définit comme un acte professionnel demandant une capacité d'analyse et de jugement. Dewey (1947) considère l'acte de penser comme un engagement exercé en contexte. Nous reviendrons sur le concept d'engagement dans le prochain point, car il représente une pierre d'assise de la démarche entreprise dans la présente recherche. Dans un avis publié par le CSÉ (2014), les formations de développement professionnel misant sur les pratiques collaboratives connaissent un engouement dans le contexte québécois. Ainsi, plusieurs formations offertes s'inscrivent dans la lignée du praticien réflexif (CSÉ, 2014). Dans cette perspective, on reconnaît que les enseignantes et enseignants ont la capacité de réfléchir dans et sur leur action et de tirer profit de leur expérience autant que des savoirs théoriques (CSÉ, 2014).

Enseigner est un acte complexe, qui se déroule dans un contexte fait d'incertitudes et d'imprévus (CSÉ, 2014). Afin dévoluer dans un tel contexte, les enseignantes et enseignants ont la possibilité de s'engager dans des démarches collaboratives afin de développer, entre autres choses, leurs compétences professionnelles. Ces dernières années, des études ont souligné l'importance de l'engagement des acteurs dans les démarches de développement professionnel (Rey, 2016 ; Savoie-Zajc, 2010). Ils deviennent les principaux agents de leur propre développement professionnel. De telles démarches représentent non pas une façon de combler un manque ou des lacunes sur des objets de formation prédéterminés, mais une façon de réfléchir à des pratiques adaptées à leur contexte. Le modèle de développement professionnel à mettre sur pied doit miser sur la compétence des acteurs, on reconnaît ainsi la capacité réflexive des enseignants et enseignantes (Giddens, 1987). Rey (2016) soutient que les formations de développement professionnel innovantes se réalisent à petite échelle, elles ne sont pas prescriptives, elles requièrent une structure souple propice aux initiatives. Cet auteur identifie la conjonction de trois aspects favorisant le développement professionnel et qui engendre un changement de pratique : 1) l'action stimulante des ressources des acteurs de référence, 2) un travail collaboratif intense, et 3) une orientation pédagogique. Bien 
que le changement de pratique ne soit pas une visée de notre projet, les aspects soulevés par Rey (2016) orientent les balises conceptuelles sur lesquelles nous souhaitons fonder notre démarche. Notre projet mise sur un espace commun pour des praticiennes et des chercheuses ${ }^{1}$ dans le but de réfléchir et de coconstruire des interventions favorisant l'apprentissage actif (AA) des élèves de maternelle 5 ans. Le modèle de développement professionnel continu retenu puise principalement dans les principes de la théorie des communautés de pratique $(\mathrm{CoP})$ telle que définie par Wenger (2005). Notre intention est d'illustrer de manière empirique, à partir de l'expérience que nous en avons vécue, les aspects prometteurs et ceux moins fructueux de cette entreprise. Autrement dit, cet article vise à mettre en relief les forces et les faiblesses que nous avons observées ainsi que celles identifiées par les enseignantes en lien avec les dispositifs mis en œuvre et inspirés du modèle de Wenger (2005). La prochaine section définit la CoP et se penche sur certaines dimensions de la théorie de Wenger (2005), dont le concept d'engagement qui représente la pierre d'assise de notre démarche de développement professionnel continu.

\section{Le cadre conceptuel : miser sur l'engagement pour entretenir une culture de collaboration au sein d'une CoP}

Wenger (2005) identifie trois principales dimensions d'une CoP : 1) l'engagement mutuel, 2) l'entreprise commune, et 3) le répertoire partagé. Nous souhaitons interpréter les dispositifs de la démarche de développement professionnel continu au regard de ces trois dimensions. Tout d'abord, l’engagement mutuel définit l'essence même de la coconstruction et de la culture de collaboration. La complémentarité des enseignantes et des chercheuses est mise à profit dans leur expertise respective pour réfléchir sur un objet commun. L’engagement qualifié de mutuel indique une relation d'interdépendance entre l'individu et le groupe. Par son appartenance et sa participation, il contribue à la négociation de sens (réfléchir sur les pratiques favorisant l'AA) et il en est également bénéficiaire. L'engagement ne se définit pas par le statut professionnel, ici le fait d’être enseignante de maternelle, il se concrétise par la participation et l'interaction dans le groupe.

Lentreprise commune assure une source de cohérence au sein de la $\mathrm{CoP}$, une cohésion résultant d'un processus collectif de négociation pour analyser les pratiques favorisant l'AA. Les membres d'une CoP partagent leurs ressources, leurs histoires, leur expérience en se penchant sur des problèmes liés à leur pratique quotidienne. La négociation de sens est reliée aux activités individuelles à partir desquelles les membres interagissent en groupe. Le sens ne peut se dissocier du contexte dans lequel il se réalise et l'interaction en groupe contribue à l'émergence de nouvelles connaissances. L'entreprise commune représente une retombée directe de ce qui est convenu de nommer le transfert de connaissances (le sens négocié sur l'AA afin de mieux comprendre comment un jeune enfant apprend). Rappelons que le propos de notre article est d'éclairer les dispositifs mis en place pour cette entreprise commune et se concentre sur la démarche.

Quant à la troisième dimension d'une CoP, soit le répertoire partagé, il comprend la production de ressources coconstruites par les membres. Ce répertoire résulte du sens partagé et des pratiques mises en œuvre grâce aux différents dispositifs proposés tout au long de la démarche. De notre point de vue, les pratiques enseignantes ne 
s'imposent pas même si elles créent des occasions de mettre au jour certaines conceptions pédagogiques qui les fondent. À ce sujet, nous expliquerons dans les résultats la production qui a été réalisée par les enseignantes d'un répertoire de vignettes d'apprentissage actif (VAA).

Tel qu'annoncé, attardons-nous au concept d'engagement qui se décline à la fois d'un point de vue individuel et collectif en prenant appui sur les travaux de Wenger (2005). Pour sengager, on doit disposer de certaines conditions. Tout d'abord, on doit évoluer dans un contexte où il est possible d'agir et d'interagir. Le concept d'engagement tel que le conçoit Wenger (2005) est notamment associé à l'idée de «se mettre en projet ». L’engagement individuel, pour sa part, invite à la mobilisation, à l'appropriation d'un projet que l'on fait sien et qui engendre un processus transformateur consistant à donner du sens à l'expérience. L'engagement d'un point de vue collectif apparaît, quant à lui, comme un élément constitutif d'une communauté de pratique (CoP). À ce sujet, on en conviendra, la participation des membres ne signifie pas nécessairement une adhésion à un projet commun. L’engagement émerge d'un sens partagé à partir des interactions entre acteurs. C'est par la négociation du sens que l'engagement collectif façonne et transcende l'engagement individuel. La dimension sociale représente un fondement de l'apprentissage chez Wenger (2005).

Avant d’aborder la méthodologie, traçons à grands traits deux autres notions de la théorie de Wenger (2005). Lauteur explique que le fait de partager autour de pratiques donne lieu à un processus qui comprend deux aspects complémentaires : 1) la participation et 2) la réification. La participation se définit par l'action de prendre part à une activité en relation avec d'autres personnes. La personne devient élément constitutif de la communauté en transcendant son expérience individuelle pour en négocier le sens en groupe. Dans la présente CoP, comme il s’agit d'enseignantes œuvrant à léducation préscolaire, nous avons choisi de qualifier en abrégé notre communauté de pratique au préscolaire la "CoPP ». La réification se définit par le fait de traiter une idée, une abstraction, un concept, comme quelque chose de tangible. Cette notion est essentielle dans la perspective du modèle de $\mathrm{CoP}$ souhaité, la réification contribue au processus transformateur. La réification consiste à donner une forme à l'expérience. Elle agit comme la résultante d'une idée transformée concrètement pour donner du sens à sa propre expérience à partir de la négociation en groupe. La variété des expériences et les interactions engendrent une légitimité et une valorisation de nouvelles pratiques.

Nous portons un regard a posteriori sur les dispositifs élaborés au fil des trois années en fonction des contextes des participantes et de leurs conceptions de l'apprentissage actif (AA). Lanalyse, essentiellement descriptive, met l'accent sur les effets des dispositifs en prenant comme cadre interprétatif les trois dimensions suivantes : 1) l'engagement mutuel, 2) l'entreprise commune, et 3) le répertoire partagé, de Wenger (2005). La prochaine section apporte quelques précisions sur l'analyse afin de documenter cet aspect et retrace les grandes étapes du déroulement du projet. 


\section{La méthodologie : proposer différents dispositifs pour soutenir l'engagement}

Telle que définie précédemment, la CoPP que nous avons constituée se veut à la fois une approche de développement professionnel continu (un modèle structurant évalué tout au long de la démarche) et un lieu d'investigation sur les stratégies favorisant l'AA. Dans le cadre de cet article, il sera question principalement du modèle proposé pour développer une culture de collaboration. En réponse à la problématique décrite, nous souhaitons identifier les conditions favorisant l'engagement des enseignantes dans une démarche de développement professionnel continu et celles qui peuvent s'avérer un frein, voire les forces et les limites des dispositifs mis en ouvre en lien avec le modèle de formation professionnelle continue retenu.

La recherche-formation, d'une durée de trois ans, a été subventionnée dans le cadre d'un programme nommé Chantier 7 soutenu par le ministère de l'Éducation, du Loisir et du Sport du Québec (MELS) (Gouvernement du Québec, 2011). Ce programme s'adressait aux universités intéressées à mettre en ouvre des projets de formation continue en partenariat avec le milieu scolaire. La collaboration devait s'instaurer dès la conception du projet et se poursuivre tout au long de sa mise en œuvre. La conseillère pédagogique responsable du dossier préscolaire à la commission scolaire a été sollicitée dès le départ, lors de la rédaction de la lettre d'intention. Quand le projet a été subventionné, elle a collaboré à toute la démarche. Léquipe de formatrices était composée de deux chercheuses, deux assistantes de recherche et de la conseillère pédagogique. Douze enseignantes ont été recrutées selon les trois critères suivants : participation sur une base volontaire, diversité des expériences selon le nombre d’années à l'éducation préscolaire et intérêt pour l'objet d’investigation, soit l’AA.

Le projet est intitulé "Comment rendre l'enfant actif dans ses apprentissages : des enseignantes du préscolaire se penchent sur la question ». Le tableau 1 présente un aperçu des visées de chacune des années en lien avec chacune des trois dimensions du modèle de Wenger (2005), des objectifs spécifiques poursuivis ainsi que des dispositifs mis en œuvre au cours des trois années du projet afin de soutenir l'engagement des enseignantes dans leur développement professionnel continu et développer une culture de collaboration. Dans la section qui suit, nous présentons pour chacune des trois années, les moments critiques et les moments porteurs ou rassembleurs à partir des dispositifs mis en œuvre. 


\section{Tableau 1}

Dispositifs mis en auvre durant les trois années du projet en lien avec les trois dimensions du modèle de Wenger (2005)

\section{Visées en lien avec le modèle de Wenger (2005)}

\section{An 1}

Réfléchir et expliciter ses conceptions de l'AA pour s'approprier une entreprise commune

\section{Objectifs spécifiques}

1. Identifier les conceptions initiales

2. Partager nos pratiques

3. Réfléchir sur la démarche de l'enfant
Dispositifs

- Quatre journées de rencontre collective

- Carnet de traces et évaluation formative

- Livre de référence

- Experts invités et activités participatives

\section{An 2}

Coconstruire des projets individuels d'intervention favorisant l'AA pour alimenter un engagement mutuel
1. Se mettre en projet

2. Documenter l'impact des interventions

3. Prendre confiance dans sa pratique professionnelle
- Planification de projets individuels

- Observation et accompagnement en binôme

- Visites dans la classe de collègues

- Présentation en CoPP des projets individuels

\section{An 3}

Rendre compte de l'AA et évaluer l'impact de ses interventions pour élaborer un répertoire partagé
1. Observer les enfants en action

2. Rédiger des vignettes d'apprentissage actif (VAA)

3. Partager avec les collègues
- Proposition d'un canevas pour les VAA

- Rétroaction et soutien sur la rédaction

- Rencontres en binômes et comptes rendus

- Bilan final en CoPP

Une précision s'impose sur l'analyse inductive qui se concentre autour des trois dimensions du cadre conceptuel et qui ont orienté les trois années du projet : l’entreprise commune, l’engagement mutuel et le répertoire partagé. Bien que l'intention de documenter les effets structurants du modèle retenu (la CoP) fût prévue dès le départ du projet, l'objet de recherche, l'AA, représente le cœur de la démarche d'analyse et a déjà fait l'objet d'autres communications (Larouche, Biron et Vaillancourt, 2017). A posteriori, il nous est apparu que les données collectées tout au long 
de la démarche par le recours à des observations, des questionnaires, des entrevues, des comptes rendus et un bilan final, nous invitaient à porter un regard rétrospectif sur l'impact des dispositifs proposés tout au long des trois années. Les résultats descriptifs que nous exposons dans cet article représentent un regard critique sur ces dispositifs en dialogue avec les trois dimensions du cadre conceptuel.

\section{Les résultats : identifier des moments critiques et des moments porteurs ou rassembleurs}

La première année du projet visait à réfléchir et à expliciter les conceptions de l'apprentissage actif des enfants (AA) des membres de la CoPP. Quatre journées de rencontre collective ont été élaborées en collaboration avec la conseillère pédagogique afin de négocier le sens de cette entreprise commune. Lors de la première rencontre, les enseignantes ont discuté de leur fonctionnement par ateliers en classe, une pratique largement utilisée en maternelle et qui représentait loccasion de mettre au jour leur conception initiale. Elles ont partagé leur compréhension des aspects favorisant l'apprentissage de leurs élèves. Un livre de référence, Partager le plaisir d’apprendre de Hohmann, Weikart, Bourgon et Proulx $(2007)^{2}$, a été proposé par les chercheuses afin d'alimenter la réflexion et la discussion. Dans cet ouvrage, les auteurs exposent de manière pédagogique leurs fondements de l'AA. Également, à chacune des rencontres de la première année, des personnes reconnues pour leur expertise en éducation préscolaire ont été invitées afin d'alimenter la réflexion autour de l'AA et des activités participatives étaient proposées aux participantes dans le but de faciliter les échanges en petites équipes. Rappelons que c’est par cette négociation de sens partagé que les membres d'une CoP s'approprient le projet, mettent en commun leur expertise individuelle et s'approprient lentreprise commune.

Afin de s'assurer que le contenu de ces rencontres répondait aux attentes des participantes et dans le but de soutenir leur engagement, celles-ci étaient invitées à remplir un petit questionnaire à la fin de chaque rencontre. Ce questionnaire investiguait les aspects suivants : ce que je retiens, mon coup de cœur, une proposition pour la prochaine rencontre, ce que j’aurais le goût d'essayer dans ma classe. Un ajustement de notre démarche a ainsi été possible d'une rencontre à l'autre afin d'adapter le contenu aux besoins et aux intérêts des membres de la CoPP. Par ailleurs, les réponses aux questionnaires ont permis d'identifier certains indicateurs de leur engagement : elles ont mentionné vouloir partager leurs coups de cœur sur des albums jeunesse, elles ont proposé de créer une plateforme électronique afin de déposer des documents intéressants pour partager leurs idées et elles ont également souligné leur appréciation de la place faite aux échanges. Enfin, mentionnons leur appréciation du carnet de traces qui leur permettait de noter leurs réflexions, leurs questions, les idées qu’elles souhaitaient mettre à lépreuve.

Parmi les éléments porteurs ou rassembleurs de cette première année, les formatrices ont observé lors des échanges que l'ouvrage de référence proposé a pu soutenir la conceptualisation de l'AA, et ce, en permettant de l'adapter au contexte d'action des participantes. Si l'on en juge par le temps de prise de parole des participantes, l'engagement s'est notamment manifesté au fil des quatre rencontres collectives par des échanges de plus en plus longs consacrés à partager les pratiques mises en œuvre entre les rencontres et les effets observés. Ces moments déchanges ont pu contribuer à créer un contexte dynamique propice à l'exploration. Tout en participant à une prise de recul sur 
leur pratique, ces discussions empreintes d'ouverture et sans jugement ont certainement soutenu un fort sentiment d’appartenance et le sens de l'entreprise commune au sein de la CoPP.

D’un point de vue critique, bien que le dispositif d'un livre de référence représente un choix pédagogique judicieux, certaines participantes ont nommé explicitement leur déstabilisation lors des discussions sur les extraits de lectures proposés. Il ressort de cela que ces lectures les ont amenées à sortir de leur zone de confort et ont entrainé une certaine résistance. Malgré la pertinence des contenus abordés par les experts invités, qui ont été appréciés tel que mentionné dans les fiches, il apparaît que cette diversité a engendré une compréhension floue de la démarche d’AA. Le tableau 2 résume les principales observations des formatrices et les principaux commentaires exprimés par les participantes en lien avec les forces et les limites des dispositifs prévus lors de l’an 1 de la CoPP qui consistait à mettre en place la première dimension du modèle de Wenger (2005), soit l’entreprise commune.

\section{Tableau 2}

\section{An 1 - L'entreprise commune}

Aspects positifs, obstacles ou difficultés des dispositifs mis en ouvre

\begin{tabular}{|c|c|c|}
\hline Visée de l'entreprise commune & $\begin{array}{c}\text { Indices positifs observés et } \\
\text { exprimés }\end{array}$ & $\begin{array}{l}\text { Obstacles ou difficultés } \\
\text { observés ou exprimés }\end{array}$ \\
\hline $\begin{array}{l}\text { L'entreprise commune assure } \\
\text { une source de cohérence au } \\
\text { sein de la CoPP, une cohésion } \\
\text { résultant d'un processus } \\
\text { collectif de négociation } \\
\text { pour analyser les pratiques } \\
\text { favorisant l'apprentissage actif } \\
\text { (AA). }\end{array}$ & $\begin{array}{l}\text { - Appropriation du livre de } \\
\text { référence } \\
\text { - Contexte dynamique, } \\
\text { propice à l'exploration } \\
\text { - Satisfaction exprimée } \\
\text { - Ouverture, sans jugement } \\
\text { - Renforcement d'un } \\
\text { sentiment d'appartenance }\end{array}$ & $\begin{array}{l}\text { - Déstabilisation par } \\
\text { la lecture du livre de } \\
\text { référence } \\
\text { - Résistance à de nouvelles } \\
\text { façons de faire } \\
\text { - Compréhension floue de la } \\
\text { démarche d'apprentissage } \\
\text { de l'enfant (l'AA). }\end{array}$ \\
\hline
\end{tabular}

La deuxième année de la CoPP visait la coconstruction de projets individuels pour favoriser l'AA. Le terme de coconstruction mérite quelques précisions. Après la première année, au cours de laquelle un ouvrage de référence a été proposé afin d'assurer une plus grande cohérence à l'entreprise commune de notre CoPP, il nous fallait faire un pas de plus. La construction de sens se fonde ici sur l'interaction, d'où le préfixe « co ». Cette interaction vise aussi bien les échanges entre collègues que l'accompagnement avec les formatrices. Selon la théorie de Wenger (2005), le sens est affaire de négociation et nous avons établi qu'un changement de pratique ne s'imposait pas. C'est de l'intérieur de leur contexte d'action que l'engagement des participantes serait favorisé pour réifier l'entreprise commune et donner forme à leur expérience. Pour ce faire, quatre dispositifs ont été prévus lors de la deuxième année : 1) la planification d'un projet personnel, 2) un accompagnement en binôme par l'équipe de formatrices (les binômes ont été pairés de manière aléatoire), 3) un calendrier de visites dans la classe de collègues et 4) une présentation des projets en CoPP. L'accompagnement et la visite dans la classe d'une collègue représentent 
deux éléments clés qui ont soutenu l'engagement mutuel des participantes.

Pour documenter les retombées de ces dispositifs et favoriser l'engagement mutuel, un entretien individuel a été mené à la fin de l'an 2. L'engagement mutuel implique que l'on s'intéresse à ce que les participantes font. Nous souhaitions les entendre sur leur projet et sur leur participation à la CoPP. Afin d'identifier les éléments porteurs ou rassembleurs ainsi que ceux plus critiques, nous avons analysé le contenu des entretiens menés à la fin de l'an 2. Ceux-ci ont été menés sous forme d'entrevue semi-structurée autour de trois aspects : 1) un retour sur les intentions de la CoPP, 2) les retombées auprès des enfants et 3) leurs propres apprentissages. Pour fins d'analyse, deux questions ouvertes sont retenues : 1) Depuis deux ans, tu participes à notre CoPP, qu'est-ce que cela t'a apporté ? 2) Qu'est-ce qui a changé dans ta pratique?

Un constat fait l'unanimité chez les participantes, elles apprécient fortement apprendre les unes des autres : «se former à même d’autres collègues ", " on s'est contaminées », " je suis enrichie des idées des autres ", "le partage de nos différentes pratiques », « ça nous fait tellement réfléchir et cheminer, partager notre vécu... voir comment elles s'y prennent ». Faire une large place au partage d'expérience renforce indubitablement l'engagement et la confiance dans son agir. L'ouverture à l'autre engendre une reconnaissance de sa singularité tout en s'exposant avec authenticité. Dans cette veine, la visite en classe, que ce soit d'accueillir une collègue dans sa classe ou se rendre dans la classe d'une collègue, représente un moment significatif pour réfléchir sur ce qui fonde son agir. L'interaction est loccasion de clarifier sa pratique. En se comparant, elles prennent conscience de leurs ressemblances et de leurs différences, et surtout - elles sont nombreuses à le nommer explicitement - elles se rassurent sur leur pratique : "ça m’a rassurée, ça m’a aussi déstabilisée, ça m’a fait évoluer », "j’ai fait des essais qui n’ont pas fonctionné, mais je me suis permis de reculer, de m’ajuster », "ça m’a fait tellement de bien d’entendre qu’elle aussi était insécure, une enseignante qui est pourtant expérimentée », «la CoPP m’a permis d’aller au fond des choses et de confirmer que ma vision était bonne ».

En plus de l'interaction comme noyau central de l'engagement mutuel, les participantes ont entrepris une démarche concrète dans leur classe pour négocier le sens de l'AA. L'expérience entraîne une meilleure compréhension et une transformation de l'action (penser et agir). Les différentes façons de s'engager traduisent différents niveaux de participation. Pour certaines, le pairage en binôme et l'accompagnement ont offert un soutien bienvenu : «le côté équipe a amené une rigueur à tenir mon projet chaque semaine », "les fois où les accompagnatrices sont venues dans ma classe, ceétait très aidant, ça m’a donné l'élan pour partir comme il faut, j’étais comme bloquée, elles sont venues au bon moment». Un grand souci du respect du rythme de chacune a teinté l'accompagnement : "c’était à mon rythme aussi avec mon contexte de classe », "vous - les accompagnatrices - nous poussiez plus loin avec des défis différents pour chacune». Se mettre en projet nécessite une prise de risque pour sortir de sa zone de confort ce qui ressort par exemple des propos tenus lors de l'entretien : "ça nous fait essayer d'autres choses en classe et ça fait réfléchir sur la façon dont les enfants apprennent », "jessaie de sortir de ma zone de confort tranquillement ».

Pour plusieurs participantes, l'entretien a été l’occasion de nommer que leur participation à la CoPP est arrivée à 
point nommé dans leur trajectoire professionnelle. Ces effets apportent une nouvelle compréhension empirique au concept d'engagement que nous souhaitons documenter. Les propos recueillis sont éloquents : « jétais dans un down, je tournais en rond, en remise en question... la flamme est rallumée », « je ne réalisais pas trop le fait dềtre toute seule au préscolaire dans mon école », « je voulais approfondir le programme et le maitriser, je veux finir ma carrière en maternelle », « ça répondait à ce que je cherchais, à un questionnement que j’avais », « il me reste 10 ans, javais le goût d’essayer de nouvelles choses».

Malgré l'effet de groupe, le soutien des pairs et l'accompagnement qui entraînent l'adhésion à la démarche, le niveau dengagement individuel est très variable. Parmi les douze participantes, trois dentre elles mentionnent des difficultés ou des réserves : « jai senti un peu de réticence de la part de certaines, parfois ça prend du temps ", « il y a beaucoup de choses dont javais entendu parler ou que javais essayées », " pour la CoPP, je ne sais pas quoi dire, quest-ce que les autres veulent savoir ? Je n’avais pas le goût d’expliquer ce que jai fait ». Pour cette dernière participante, tout au long de l'entretien, en dépit des efforts répétés pour faire expliciter concrètement le projet réalisé, elle formulera surtout des exemples spécifiques autour délèves ayant des besoins particuliers. Il est possible que la difficulté à expliciter sa pratique relève, dans ce dernier cas, du fait qu'elle ne s'est pas vraiment mise en projet. Toutefois, son engagement s'est traduit par une présence assidue et une participation quelle qualifie de plus personnelle. Une mise en garde s'impose dans ce que nous qualifions ici comme un moment critique. L’engagement mutuel ne s'impose pas, celui-ci se développe quand les membres d'une CoPP réifient et en font leur lentreprise commune. Il apparait que les différents niveaux d’engagement dont nous témoignons ici rendent compte de la liberté d’adhésion à la démarche que nous ne souhaitions pas imposer.

Quant au dernier dispositif proposé, celui qui vient clore cette deuxième année par la présentation des projets, nous y prêtons une interprétation plus mitigée. En effet, les propos de quelques participantes laissent croire à un éparpillement dans la diversité des projets présentés : «jai trouvé que les présentations nétaient pas en lien », «jétais nerveuse de présenter, mais j’ai vu que d'autres aussi ont fait complètement autre chose, comme si on était libres de faire ce qu’on veut ». Par ailleurs, elles étaient réticentes à présenter : « finalement jai aimé faire ma présentation même si au départ ça ne me tentait pas, ça m’a forcée à me recentrer », «mon but nétait pas de présenter un projet pour épater la galerie ». Ces remarques recueillies en entretien individuel témoignent que ce dispositif représente un moment critique de la démarche. Nous observons qu’aucun commentaire n’a été formulé lors des présentations en CoPP. L'évitement de la confrontation est-il un signe de respect ou une peur du jugement ? Chose certaine, en conviant les participantes à présenter leur projet nous les invitions à poser un regard analytique sur celui-ci. Audelà du niveau descriptif visant à rendre compte de ce qui a été réalisé, c’est sur leur capacité réflexive à porter un regard distancié sur l'entreprise commune que ce dispositif a porté fruit. La responsabilité assumée de se mettre en projet vient ainsi consolider le sentiment d'appartenance de la CoPP et une meilleure compréhension de l'AA. Le tableau 3 qui suit résume les principaux aspects observés par les formatrices ou explicités par les participantes en lien avec des moments porteurs et des moments critiques au regard des dispositifs prévus lors de l'an 2 de la CoPP. Ces éléments sont en lien avec la deuxième dimension issue des travaux de Wenger (2005), soit l'engagement 
mutuel.

Tableau 3

An 2 - L'engagement mutuel

Aspects positifs, obstacles ou difficultés des dispositifs mis en œuvre

\begin{tabular}{|c|c|c|}
\hline Visée de l'engagement mutuel & $\begin{array}{c}\text { Effets positifs observés ou } \\
\text { exprimés }\end{array}$ & $\begin{array}{c}\text { Difficultés observées ou } \\
\text { exprimées }\end{array}$ \\
\hline $\begin{array}{l}\text { Les participantes s'engagent } \\
\text { et se mettent en projet. Non } \\
\text { seulement elles adhèrent } \\
\text { à l'entreprise commune, } \\
\text { mais elles contribuent à la } \\
\text { recherche de solution de par } \\
\text { leur projet individuel. }\end{array}$ & $\begin{array}{l}\text { - Partage d'expérience } \\
\text { - Individualité reconnue } \\
\text { - Respect du rythme } \\
\text { - Responsabilité assumée }\end{array}$ & $\begin{array}{l}\text { - Éparpillement, diversité } \\
\text { - Différents niveaux de } \\
\text { participation } \\
\text { - Difficulté à expliciter sa } \\
\text { pratique } \\
\text { - Évitement de la } \\
\text { confrontation }\end{array}$ \\
\hline
\end{tabular}

L'an 3 concerne l'évaluation de l'impact des interventions favorisant l'AA. Alors que l'an 2 a permis de dégager comment les actions individuelles sont au service de l’entreprise commune, cette troisième année misait sur la constitution d’un répertoire partagé. Wenger (2005) explique que le répertoire partagé combine les éléments de réification et de participation pour rendre compte des interprétations des membres de la CoPP. Le répertoire les amène à produire des réalisations qui concrétisent l'entreprise commune, à consolider l'engagement mutuel et une façon de se donner des ressources dont ils vont tirer profit. Le répertoire reflète les façons particulières, mais n’impose pas une signification d’emblée. Ici encore, le sens n’est pas donné d'avance, il se négocie de l'intérieur de l'expérience. Pour produire un répertoire, un dispositif a été proposé aux participantes : la rédaction de vignettes d’apprentissage actif (VAA) dont il sera question plus loin. Ce dispositif nous semblait répondre à la double finalité d'un effet structurant pour élaborer un répertoire partagé tout en faisant appel à la compétence des participantes pour rendre compte de manière contextualisée des pratiques favorisant l’AA. En cohérence avec la perspective compréhensive et interprétative endossée par notre équipe de recherche, ce dispositif se voulait suffisamment souple et adapté aux différents contextes des participantes. Par ailleurs, la conseillère pédagogique voyait la rédaction des VAA comme un outil utile et convivial de consignation des observations sur les démarches des enfants et un moyen pertinent de communication aux parents. Ce dispositif a toutefois été perçu par plusieurs participantes comme une contrainte et elles ont manifesté plusieurs réticences dans l'exploitation qui en a été faite tout au long de l'année. Avant d’en dégager les avantages et les limites, voyons plus en détail ce que nous avions prévu par ce dispositif.

En début de l'an 3, lors de la première rencontre de la CoPP, une proposition a été présentée par l'équipe de formatrices d'élaborer un répertoire à partir de vignettes d’apprentissage actif (VAA). Ce dispositif s'inspire des travaux de Margaret Carr (2001, Carr et Lee, 2012) sur les « learning stories ». En quelques mots, voici ce dont il 
est question quand on parle de vignettes. Les VAA sont de courtes descriptions accompagnées de photos d'un ou de quelques enfants en action dans une situation précise en interaction avec l'environnement. Elles permettent de décrire et de reconnaître l'apprentissage qui se déroule à travers le jeu ou dans l'activité. Les VAA montrent comment l'enfant est « en train d'apprendre à apprendre ».

Plus précisément, nous souhaitions à l'aide de ce dispositif insister sur la démarche d'apprentissage des enfants ${ }^{3}$. Lors d'une rencontre en CoPP, les participantes ont pu se familiariser avec ce dispositif à l'aide d'un canevas qui leur a été proposé. Sur place, elles ont pu s'exercer à partir de trois photos prises dans leur milieu respectif. La rédaction de vignette se réalise en six étapes. La première étape concerne une mise en contexte pour expliquer l'activité dans laquelle l'enfant est engagée. La deuxième décrit la démarche de l'enfant en lien avec les photos. La troisième concerne l'interprétation de l'enseignante. La quatrième suggère des réinvestissements possibles pour soutenir l'enfant dans ses apprentissages. La cinquième livre quelques commentaires qui ont été recueillis auprès de l'enfant, des pairs, des collègues ou des parents. Enfin, à la sixième étape, il s'agit de trouver un titre évocateur, car il est souvent plus facile à trouver à la fin de la rédaction.

La figure 1 illustre un exemple visuel du format d'une VAA. Pour les premières VAA qu'elles ont eu à rédiger dans le mois suivant la rencontre, une rétroaction a été acheminée par une assistante de recherche à chacune des participantes pour les aider à étayer leur description. Elles devaient rédiger deux VAA par mois pendant trois mois. Trois pairages en binôme étaient prévus au calendrier pour qu’elles se présentent mutuellement leur VAA. Afin de les soutenir plus étroitement dans cette analyse réflexive, une formatrice s'est associée à chacune de ces rencontres en binôme. 


\begin{tabular}{|l|l|}
\hline Un titre : Un mariage très spécial \\
\hline Une mise en contexte : J'ai mis à la disposition des enfants des foulards aux couleurs variées lors des \\
ateliers libres.
\end{tabular}

Figure 1- Exemple visuel d'une VAA

D’un point de vue empirique, le répertoire représente un matériau de recherche très riche pour analyser la conception de l'apprentissage actif du point de vue des enseignantes, entre autres, sur le rôle qu'elles exercent pour favoriser l'apprentissage de leurs élèves. Par contre, nous constatons une limite : le fait de prendre les enfants en photo a peut-être influencé les participantes en posture d'extériorité. Dans les VAA, elles témoignent de l'action de l'enfant et non de leurs pratiques pour soutenir l'AA.

Les retombées de ce dispositif sont nombreuses sur le plan du développement professionnel et d'une pratique réflexive sur l'AA. Tout d'abord, les VAA constituent un outil relativement simple de consignation et d'évaluation des apprentissages des élèves. Les participantes ont su saisir des situations authentiques d'apprentissage qui mettent 
en valeur les " dispositions » de l'enfant. Elles mentionnent que le choix des situations les a incitées à observer certains enfants qui passent souvent inaperçus dans leur groupe. De plus, elles ont pu identifier les forces de leurs élèves et garder des traces de leur évolution. Dans les étapes de rédaction, notamment celle portant sur les réinvestissements possibles, cela leur permettait de projeter des défis adaptés aux élèves ciblés afin de nourrir leur motivation.

Par ailleurs, ce dispositif a provoqué de nombreuses résistances chez les participantes. Une première réaction s'explique par le rapport à l'écrit. Alors que nous nous attendions à constituer un répertoire d'au moins une soixantaine de VAA, le répertoire partagé comprend seulement 35 situations. Il a fallu diminuer nos attentes des deux vignettes mensuelles à une seule par mois. Lors du bilan, les participantes ont mentionné que la sélection de la situation, la prise de photos sur le vif et la rédaction de la vignette était une entreprise très exigeante. Cette tâche leur a demandé plus de temps que celui qui leur était alloué. Elles ont préféré se concentrer sur une seule situation et en discuter plus en profondeur en binôme. De leur point de vue, il est plus facile d'expliquer oralement la démarche d'un enfant plutôt que d'avoir à la mettre par écrit. Ces rencontres ont suscité des interactions stimulantes entre pairs et avec les formatrices. Dans le bilan que nous dressons du dispositif, il apparaît clairement qu'un accompagnement étroit est nécessaire pour en permettre l'appropriation parce que cela exige de développer une certaine aisance à décrire le contexte, les actions de l'enfant et l'interaction qui émerge de la situation décrite. Livrées à elles-mêmes, les participantes ont plus de difficulté à analyser et à interpréter la démarche de l'enfant en vue d'en faire une synthèse. Le questionnement d'une collègue et de la formatrice encourage, en outre, une description plus fine dans l'analyse pour nommer les stratégies et le processus d'apprentissage de l'enfant. Le tableau 4 résume les grandes lignes des moments porteurs et des moments critiques au regard du dispositif prévu lors de l'an 3 de la CoPP en lien avec le répertoire partagé sous forme de VAA. 


\section{Tableau 4}

An 3 - Le répertoire partagé

Aspects positifs, obstacles ou difficultés des dispositifs mis en ouvre

\begin{tabular}{|c|c|c|}
\hline $\begin{array}{c}\text { Visée du répertoire } \\
\text { partagé }\end{array}$ & $\begin{array}{c}\text { Avantages observés et } \\
\text { exprimés }\end{array}$ & Limites observées et exprimée \\
\hline $\begin{array}{l}\text { Le répertoire partagé } \\
\text { comprend la production } \\
\text { de vignettes (VAA) } \\
\text { pouvant être réinvesties } \\
\text { dans les milieux scolaire } \\
\text { et universitaire. }\end{array}$ & $\begin{array}{l}\text { - Matériau riche de situations } \\
\text { authentiques } \\
\text { - Observation de situations } \\
\text { concrètes } \\
\text { - Attention portée à certains } \\
\text { enfants plus discrets } \\
\text { - Interactions stimulantes } \\
\text { entre pairs } \\
\text { - Accès aux conceptions } \\
\text { individuelles } \\
\text { - Réflexion sur l'action } \\
\text { - Légitimation et valorisation } \\
\text { exprimées } \\
\text { - Motivation observable chez } \\
\text { les enfants }\end{array}$ & 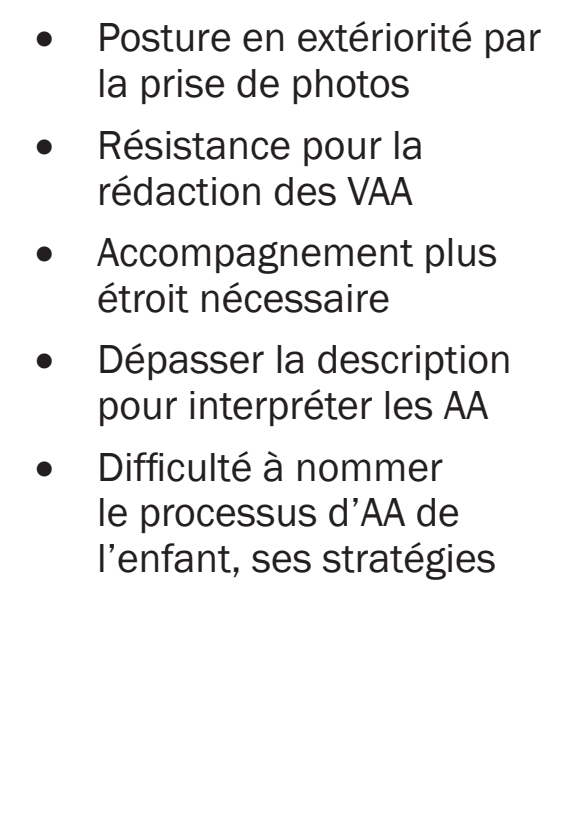 \\
\hline
\end{tabular}

\section{Conclusion : nourrir une culture de collaboration en soutenant l'engagement}

La démarche de développement professionnel continu de trois ans que nous venons de décrire et qui s'est vécue auprès de douze enseignantes du préscolaire montre notamment l'importance de l'engagement des enseignantes au sein d'une CoPP. Nous avons fait part de certains dispositifs qui convergent sur l'importance de l'engagement des acteurs de terrain comme facteur clé de l'évolution des pratiques (Rey, 2016). Ainsi, c'est en plaçant les enseignantes comme principal agent de leur propre développement professionnel, c'est-à-dire en favorisant les échanges sur leur pratique et l'expérimentation de nouvelles, qu'elles acquièrent la confiance et le regard critique sur leur agir. Par contre les trois dimensions issues du cadre de Wenger (2005), l'engagement mutuel, l'entreprise commune et le répertoire partagé, nous incitent à porter un regard critique sur notre propre démarche et sur le rôle exercé au sein de la CoPP. Est-ce que ce cadre que nous voulions souple et respectueux des pratiques a atteint ce but ? Rappelons que la CoPP ne visait pas à combler un manque ou des lacunes, mais s'inscrivait dans un effort pour affirmer et consolider l'acte d’enseigner en misant sur leur capacité réflexive.

La collaboration entre praticiens et chercheurs prend parfois une forme prescriptive, top-down. L'expérience de pratique ne prend souvent sa légitimité, dans la culture de formation professionnelle à l'enseignement, quen tant qu'elle constitue ou devrait constituer un lieu d’application de savoirs prescrits (Desgagné et Larouche, 2010, p.7). 
Ces auteurs conçoivent la recherche collaborative comme une entreprise de légitimation du savoir d'expérience qui offre aux praticiens un espace dit réflexif pour faire entendre leur voix. En ce sens, bien que singulière et documentée a posteriori, la démarche dont nous voulions rendre compte dans cet article veut contribuer à cette perspective collaborative, un fort désir de rapprochement entre milieux de pratique et de recherche. Une limite importante réside dans la difficulté à se distancier de nos propres pratiques de recherche et nécessiterait un effort de théorisation pour en valider la rigueur scientifique. Toutefois, force est de constater que non seulement les participantes ont été actives dans les échanges et ont remis en cause leur pratique, il en fut de même pour les personnes formatrices-chercheuses engagées dans le processus. Bien que cet aspect puisse paraître inhérent à une CoPP, il convient de souligner combien cela peut être exigeant pour la personne formatrice parce que cela exige notamment de mettre parfois de côté des savoirs issus de la recherche et qui peuvent entrer en conflit avec certains propos ou points de vue issus de l'expérience singulière d'une classe. Bref, cette question gagnerait certainement à être approfondie et mieux documentée, car il s'est avéré précieux et enrichissant, voire selon nous essentiel, que tous les acteurs du projet s'ajustent et remettent en question leurs convictions tout au long de la démarche afin de nourrir la réflexion et ne pas imposer une vision particulière de l'AA. À ce sujet, la capacité réflexive des formatrices afin de s'ajuster et de s'adapter lors de la mise en œuvre des différents dispositifs s'est manifestée à différentes occasions, comme dans les moments d'écoute et de soutien individuel, ou encore en allant rencontrer les enseignantes dans leur milieu afin de mieux saisir leur réalité. En terminant, rappelons que le développement professionnel continu défini comme un véritable acte professionnel demandant une capacité d’analyse et de jugement exercé en contexte a aussi le potentiel de contribuer au développement de l'identité professionnelle, tel que le soutient Wenger (2005). 


\section{Références}

Beaumont, C., Lavoie, J. et Couture, C. (2011). Les pratiques collaboratives en milieu scolaire : cadre de référence pour soutenir la formation. Québec, QC : Université Laval, Centre de recherche et d'intervention sur la réussite scolaire (CRIRES).

Campbell, C., Osmond-Johnson, P. et Faubert, B. (2016). The state of educators professional learning in Canada. Oxford, OH : Learning Forward.

Carr, M. (2001). Assessment in early childhood settings. Learning stories. Londres, Royaume-Uni : Sage.

Carr, M. et Lee, W. (2012). Learning stories. Constructing learner identities in early education. Londres, Royaume-Uni : Sage.

Conseil supérieur de léducation (2014). Le développement professionnel, un enrichissement pour toute la profession enseignante. Québec, QC : Gouvernement du Québec.

Dameron, S. et Josserand, E. (2007). Le développement d’une communauté de pratique : une analyse relationnelle. Revue française de gestion, 174(5), 131-148.

Desgagné, S. et Larouche, H. (2010). Quand la collaboration de recherche sert la légitimation d'un savoir d'expérience. Recherches en éducation. Hors série no 1, 7-18. http://www.recherches-en-education.net/spip.php?article130

Dewey, J. (1947). Expérience et éducation. Paris, France : Éditions Bourrelier.

Donnay, J. et Charlier, E. (2008). Apprendre par l'analyse des pratiques. Initiation au compagnonnage réflexif (2e éd.). Namur, Belgique : Presses universitaires de Namur.

Giddens, A. (1987). La constitution de la société. Paris : Presses universitaires de France.

Gouvernement du Québec (2002). Politique gouvernementale des adultes et de formation continue. Québec, QC : Ministère de l'Éducation, du Loisir et du Sport.

Gouvernement du Québec (2011). Programme de soutien à la formation continue du personnel scolaire. Guide pour l'appel de projets. Québec, QC : Ministère de l'Éducation, du Loisir et du Sport.

Hargreaves, A. et Fullan, M. (2013). The power of professional capital. The Learning Forward Journal, 34(3), 36-39.

Helterbran, V.R. et Fennimore, B.S. (2004). Collaborative early childhood professional development: Building from a base of teacher investigation. Early Childhood Education Journal, 31(4), 267-271.

Hohmann, M., Weikart, D., Bourgon, L. et Proulx, M. (2007). Partager le plaisir d’apprendre. Guide d'intervention éducative au préscolaire (2 éd.). Montréal, QC : Gaëtan Morin.

Larouche, H. Biron, D. et Vaillancourt, J. (2017). Décrire et valoriser l'apprentissage actif chez les jeunes enfants au moyen de " vignettes». Communication dans le cadre du congrès de l'OMEP et du Symposium « Les relations de la petite enfance : les fondations d'un avenir durable », Opatijia, Croatie, juin.

Li, Y.-L. (2008). Teachers in action research: Assumptions and potentials. Educational Action Research, 16(2), $251-260$.

Ouellet, S., Caya, I. et Tremblay, M-P. (2011). L’apport d'une communauté d’apprentissage de personnels scolaires. Éducation et formation, e-293, mai, 207-226.

Rey, O. (2016). Le changement, c'est comment ? Dossier de veille de l'IFÉ, 107, 1-18.

Savoie-Zajc, L. (2010). Les dynamiques d'accompagnement dans la mise en place de communautés d'apprentissage. Éducation et formation, e-293, mai, 9-20.

Wenger, É. (2005). La théorie des communautés de pratique (Trad. par F. Gervais). Saint-Nicolas, QC : Presses de l’Université Laval. 


\section{(Endnotes)}

1 Les participantes au projet sont toutes des femmes, nous adopterons le genre féminin pour rendre compte de l'échantillon.

2 Ce livre est une traduction et une adaptation des travaux du programme Highscope.

3 Ce dispositif, qui prend appui sur les travaux de Margaret Carr (2001, Carr et Lee, 2012) sur les « learning stories », fera l'objet d'une description détaillée dans une publication de nos travaux. Dans le cadre de cet article, nous souhaitons nous concentrer sur la démarche et l'impact des dispositifs. 